\title{
Zur Form der die Zeitdauer ausdrückenden Adverbiale im Tscheremissischen
}

Die zeitliche Dauer des Geschehens wird in den fiu. Sprachen u.a. durch Adverbiale ausgedrückt, die in den Objektkasus stehen. In den Sprachen, wo das Objekt regelmässig eine besondere Akkusativform hat, befindet sich auch das Adverbiale der Zeitdauer allgemein in diesem Kasus (so im Lappischen, s. z.B. Erkki Itkonen, Lappische Chrestomathie, S. 51, Konrad Nielsen, Lærebok i lappisk I, S. 321; im Ungarischen, s. z.B. A mai magyar nyelv rendszere. Leíró nyelvtan II, S. 194195; im Ungar. kann jedoch in bestimmten Sonderfällen ein suffixloses nominativisches Adverbiale auftreten, a.a.O. S. 186). In den ostseefinnischen Sprachen, die ein vielförmiges Objekt haben, wird die Form der sog. objektverwandten Adverbialbestimmungen generell nach denselben syntaktischen Regeln bestimmt wie beim eigentlichen Objekt (s. z.B. über das Finn. E. N. Setälä, Suomen kielen lauseoppi, S. 28-29; über das Estn. J. Valgma - N. Remmel, Eesti keele grammatika, S. 176; über das Weps. Lauri Kettunen, MSFOu 86, S. 124-125, 137-138).

In jenen fiu. Sprachen wiederum, wo das Objekt entweder regelmässig in der Grundform steht - so z.B. im Ostjakischen und einem Teil des Wogulischen - oder wo durch eine besondere Akkusativform nur das bestimmte Objekt ausgedrückt werden kann, während das unbestimmte Objekt in der Grundform steht - so im Mordwinischen, in den permischen Sprachen und einem Teil der wogulischen Dialekte - steht das die Zeitdauer ausdrückende Adverbiale im Nominativ (s. z.B. Wolfgang Steinitz, Ostjakische Grammatik, S. 51; Грамматика мордовских языков, фонетика и морфология, S. 107; Грамматика современного удмуртского языка, фонетика и морфология, S. 94-95; Современный коми язык I, S. 147-148; wogul. 
Belege s. z.B. Kannisto-Liimola, MSFOu 109, S. 39, Zeile 16, MSFOu 111, S. 154, Zeile 11 und S. 160, Zeile 1).

Obwohl sich das entworfene allgemeine Bild durch eine eingehende Untersuchung der einzelnen Sprachen durchaus noch differenzieren kann, lässt sich doch der Gebrauch in den genannten Sprachen in grossen Zügen in folgender Regel vereinen: das Adverbiale der Zeitdauer hat dieselbe Form wie das unbestimmte Objekt.

Von einer fiu. Sprache war oben noch nicht die Rede: dem Tscheremissischen od. Mari. Hier ist der Kasus sowohl des bestimmten als auch des unbestimmten Objekts allgemein der auf $-m$ endende Akkusativ; nur in einigen Sonderfällen, vor allem in Verbindung mit dem Verbalnomen, kann das Objekt dem Nominativ entsprechen (s. z.B. Alhoniemi, FUF XXXVIII, S. 1-136). Aufgrund der Praxis in den verwandten Sprachen würde man erwarten, dass auch bei den Adverbiale der Zeitdauer der Akkusativ Verwendung fände.

In einigen der tscherem. Grammatiken, wo überhaupt die Rede ist von der Form des Adverbiale der Zeitdauer, findet sich denn auch die Erwähnung, dass in diesbezüglichen Fällen der Akkusativ verwendet werde (s. z.B. F. J. Wiedemann, Versuch einer Grammatik der tscheremissischen Sprache, S. 26, Ernst Lewy, Tscheremissische Grammatik, S. 131-132). In Ivan Galkins historischer Grammatik wird die Verwendung des Akkusativs jedoch ausdrücklich als dialektal bezeichnet (Историческая грамматика марийского языка, морфология, S. 45); hinsichtlich der Schriftsprache fand ich weder in diesem Buch noch in einigen anderen Grammatiken der Schriftsprachen eine Erwähnung. Die umfangreiche Grammatik von Ödön Beke, Cseremisz nyelvtan, zeigt jedoch, dass es in der Sprache auch eine andere Praxis gibt: hier heisst es, das die zeitliche Dauer ausdrückende Adverbiale habe die Form des Nominativs (S. 230-231); für die Verwendung des Akkusativs hat Beke denn auch nur Beispiele für temporale Adverbiale, die auf die Frage 'wann?' antworten (S. 197).

Wer sich anhand der tscherem. Dialekttexte mit dieser Frage beschäftigt, der sieht, dass vor allem die verschiedene Vertretung in den einzelnen Dialekten zu den zweierlei Darstellungen geführt hat. Als häufigster Gebrauch erweist sich jedoch der 
von Beke genannte, dass nämlich das Adverbiale der Zeitdauer die Form des Nominativs hat. Nach den zur Verfügung stehenden Dialekttexten ist der Nominativ in allen Mundarten möglich; dass eine solche Verwendung dieses Kasus in den Grammatiken kaum erwähnt wird, wird einfach darauf beruhen, dass die verschiedenen Verwendungsweisen des Nominativs in den Grammatiken überhaupt nicht so genau geschildert werden wie die der anderen Kasus.

In den diesbezüglichen Adverbiale der Zeit scheint der Nominativ - Stichproben zufolge - dem Stand der Schriftsprachen zu entsprechen; in dieselbe Richtung weist natürlich indirekt auch Galkins obige Behauptung, die Verwendung des Akkusativs sei dialektal. Hier einige Belege aus der östlichen und westlichen Schriftsprache: $A л а-м$ ьляр жс а п киенам дыр ... (Мут орланге, 41) 'Ich habe wohl eine Zeitlang gelegen ...' |Тунемше-влак- - пашам 2 шаг а тат 30 минут ыитеныт - - (А. С. Пчёлко-Г. Б. Поляк, Арифметика 3 класслан, 128) 'Die Schüler arbeiteten 2 Stunden 30 Minuten-'//Труенам мӥнь ти землялан н ви л л ви и. (Н. Ильяков, Эдемвлӓ дӓ ивлӓ, 7) 'Ich habe mich 40 Jahre lang um dieses Land bemüht.' / $М$ аня $я$ и и в̈нде тел йӹ̈де тракторвлё, машинавлӓ доно Йыл гач стройышаш лесым иыпитат. (Ончыко 6/1961, 22) 'Wieviele Jahre haben sie nun jedes Jahr Bauholz mit Traktoren und Kraftfahrzeugen quer über die Wolga transportiert.'

Der Akkusativ ist aber auch nicht ganz unbekannt in der Schriftsprache; folgendes Beispiel stammt aus einer Märchensammlung: Hy, вате, мый адак коштын толам и $к$ арням. (Марий йомак, gesammelt von К. А. Четкарев, 102) 'Nun Weib, nachdem ich wieder eine Woche lang gewandert habe, werde ich (zurückkommen).'

Wie gesagt: der Nominativ ist auch in all jenen Dialekten möglich, für die Dialekttexte zur Verfügung stehen. Hierfür zeugt u.a. ein Beispielsatz, den Yrjö Wichmann aus fünf verschiedenen Dialekten aufgezeichnet hat: $i k \quad k e$ ( $t^{\prime} s^{\prime}$ a $\beta u$ -

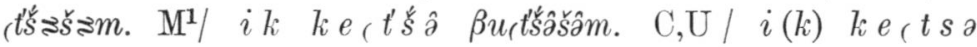

${ }^{1}$ Für die Bücher mit Dialekttexten sowie die tscherem. Dialekte werden in diesem Aufsatz dieselben Abkürzungen verwendet wie z.B. 


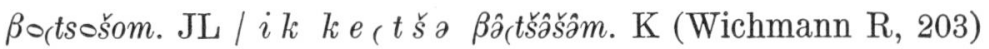
'Ich wartete einen Tag.' Noch einige weitere Belege aus anderen Mundarten: $i k-k o k$ arńa pürüm pušket kijen-da kolen. (Beke R, 46 B) 'Ein [bis] zwei Wochen lag' er Blut sprengend und starb.' / malen olda, maneš, $k \circ m \bar{\imath}--$ (Beke T I, $171 \mathrm{JT}$ ) 'Ihr habt drei Jahre geschlafen (sagt er) - -'/ $k \circ m$ djot mân denem djomolan djomoldo! (Beke T I, 589 Č) 'Bete an meiner Seite (oder: mit mir) drei Nächte zu Gott!'

In verneinten Sätzen drücken die diesbezüglichen Adverbiale der Zeit meist den Zeitraum aus, in dessen Verlauf die durch das Verb ausgedrückte Handlung gar nicht geschieht ${ }^{1}$,

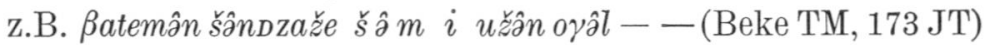
'Die Augen meiner Frau sahen [seit] sieben Jahren nicht - -'। ti em denâ o kińel $i k k e$, $t^{\prime} \xi \hat{\jmath} \delta a i k$ üt, mala. (Beke M, 470: $1 \mathrm{U})$ 'Von diesem Mittel erwacht sie nicht, schläft einen Tag und eine Nacht lang [eigtl. mit dieser Medizin erwacht sie einen Tag und eine Nacht nicht, (sondern) schläft]'. Andererseits begegnen jedoch auch Satzzusammenhänge, wo ein solches temporales Adverbiale einen Zeitraum ausdrückt, der länger ist als die durch das Verb ausgedrückte Handlung, d.h. wo die Bedeutung des Adverbiale der Bedeutung der objektverwandten Bestimmung im finnischen Verneinungssatz ent-

in meiner Arbeit Über die Form des zu Verbalnomina tretenden Objekts im Tscheremissischen, s. FUF XXXVIII. Ausserdem werden in dem vorliegenden Artikel folgende Abkürzungen verwendet:

Budenz NyK $3=$ Budenz József, Erdei cseremiszség. Nyelvtudományi Közlemények 3 .

Jevsevjev II = Timofej Jevsevjev, Volksdichtung, Band II. Manuskript im Besitz der Finnisch-ugrischen Gesellschaft, Helsinki.

${ }^{1}$ Interessanterweise haben die objektartigen Bestimmungen im Finnischen keine solche Bedeutungsaufgabe im Verneinungssatz. Die formal negative Entsprechung des positiven Satzes näin hyvin kaks $i$ - uo t ta 'ich sah gut zwei Jahre [lang]', en nähnyt hyvin kaht a - u o t t a 'ich sah gut nicht zwei Jahre [lang]' enthält an sich eine Bejahung: ich sah gut, aber eine kürzere Zeit als zwei Jahre. Falls man im Fi. einen Zeitraum ausdrücken will, in dessen Verlauf die Handlung gar nicht geschieht, muss z.B. der Illativ verwendet werden, en nähnyt hyvin kahte en ou o te en 'ich habe zwei Jahre lang nicht gut gesehen'. 


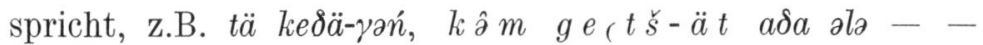
(Beke V, 156: 57-58 K) 'Wenn ihr fortgeht, werdet ihr nicht drei Tage leben - - .'

Der Akkusativ begegnet in Adverbialbestimmungen, die die Zeitdauer ausdrücken, nur in einem sichtlich begrenzten Dialektgebiet und auch dort also neben dem Nominativ. Alle Akkusativbelege, die ich in mundartlichen Texten gefunden habe, stammen nämlich entweder aus dem östlichen Teil des sog. Jaransker Dialekts (JT) oder aus einer Mundart, die in der westlichen Forschung als Dialekt von Carevokokšajsk bezeichnet wird, wozu also auch der Morker Dialekt gehört.

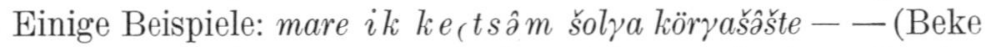
T I, 283 JT) 'Der Mann verbringt (eig. steht) einen Tag im Loch - -' / mâń mo-ńar im ôlem, tṇnar-yana moro! (Beke TT, 69: 209 JT) 'Schrei so oft, wieviel Jahre ich noch le-

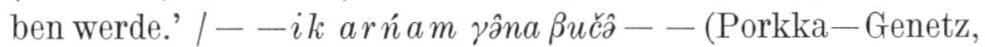
$8 \mathrm{C})$ ' - - nur eine Woche warte - -'/ kuzeyânat ikta žâ $\beta r e^{-}$ ma m ašnâza - - (Jevsevjev II, 473 C) 'Versorgt irgendwie

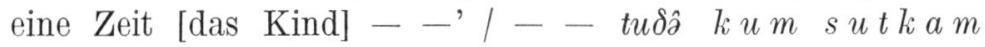
malen. (Jevsevjev II, 599 C) ' - - er schlief drei Tage und Nächte.' (S. auch И. Г. Иванов, Г. М. Тужаров, Северо-западное наречие марийского языка, S. 108.)

Unter jenen hunderten von Beispielen, die ich aus anderen Dialekttexten exzerpiert habe, befindet sich keine einzige Akkusativverwendung, die mit obigen Belegen vergleichbar wäre. Die einzigen Satzzusammenhänge, wo man vielleicht ein Adverbiale der Zeitdauer in Akkusativform vermuten könnte, liefern zwei Beispiele, in denen das Prädikat malen ertarem 'schlafend verbringen' lautet, z.B. - - malen ertarat $i k$ ü $\delta$ วิm. (Beke M, 89: 12 U) '- - sie schlafen eine Nacht durch.' Mit diesem zusammengesetzten Prädikat kann auch der Nominativ verbunden werden, z.B. küptšâk koklaš malen ertara kum $i$. (Beke M, 173: 10-11 U) 'Er schläft zwischen den Polstern drei Jahre lang.' In diesen Fällen handelt es sich jedoch äusserst deutlich darum, dass an das diesbezügliche zusammengesetzte Prädikat das die Zeit ausdrückende Wort entweder als deutliches Objekt (erstes Beispiel) oder dann als Adverbiale der Zeit (zweites Beispiel) treten kann. Mit dem 
Einzelverb ertarem 'verbringen, verbrauchen' wird ein die Zeit ausdrückendes Wort stets als Objekt verbunden, z.B. $n \bar{u}$, er lam ertaret kân - - (Beke T I, 614 Č) 'Nun wenn du den morgigen Tag überlebst _ - ', desgleichen mit dem zusammengesetzten Prädikat ertaren koltem 'verbringen (Zeit)', ein Beleg aus der westlichen Schriftsprache: $K$ илм им эртарен колтенам - - (Ончыко 6/1961, 21) 'Ich habe drei Jahre verbracht - - .'

Ein klarer Grund dafür, weshalb im Tscheremissischen die Adverbiale der Zeitdauer allgemein eine andere Form haben als die eigentlichen Objekte, dürfte sich schwer nachweisen lassen. Meinerseits möchte ich zwei Erklärungsmöglichkeiten anführen.

Man wird es zunächst nicht für ausgeschlossen halten können, dass die Verwendung im Tscherem. auf den Einfluss der Türksprachen zurückgeht. In den Türksprachen wird bekanntlich überall in den Adverbiale der Zeitdauer die der Grundform entsprechende Form verwendet (K. Grönbech, Der türkische Sprachbau I, S. 141-142), und speziell für das Wolgatatarische erwähnt Michael Brynjowśkyj, »der Indefinit - - bezeichnet die Dauer, wozu der Akkusativ nicht fähig ist» (UAJb XXIX, S. 73). Es ist jedoch anzumerken, dass in den Türksprachen gerade das bestimmte Adverbiale der Zeitdauer auch die Form des Akkusativs haben kann (s. Grönbech, a.a.O., S. 156) und dass speziell im Tschuwaschischen, wo Dativ und Akkusativ formell zusammengefallen sind, das Adverbiale der Zeitdauer auch in einigen anderen Fällen in diesem Kasus stehen kann (s. z.B. John R. Krueger, Chuvash Manual, S. 104; s. auch Maтериалы по грамматике современного чувашского языка, S. 18 , vgl. S. 15).

Andererseits kann man auch versuchen, die Verhältnisse im Tscheremissischen von der einheimischen Grundlage her zu erklären. Wie z.B. zu Beginn dieser Darstellung erwähnt wurde, wird in vielen fiu. Sprachen, u.a. in den dem Tscheremissischen am nächsten stehenden, das bestimmte Objekt durch die Akkusativ-, das unbestimmte durch die Grundform ausgedrückt. Es dürfte nicht ganz unmöglich sein, dass das Tscherem. einmal denselben Stand vertreten hat. Falls dem so gewesen wäre, 
könnte sich in der Nominativform des Adverbiale der Zeitdauer gut eine Erinnerung an das alte unbestimmte Objekt erhalten haben. Dass der Akkusativ in den Adverbiale der Zeitdauer bei einem Teil des Sprachraumes in einigen Fällen den Nominativ verdrängt hätte, ist leicht zu verstehen; wie beispielsweise die Belege von malen ertarem 'schlafend verbringen' zeigen, ist der Unterschied zwischen Objekt und temporalem Adverbiale von Fall zu Fall recht geringfügig.

Ausser durch Formen, die zum eigentlichen Kasussystem gehören, wird die zeitliche Dauer des Geschehens im Tscheremissischen jedoch noch durch andere Mittel ausgedrückt. Von den sonstigen in Frage kommenden Formen interessieren die auf -šken ausgehenden vielleicht am meisten. Den Dialekttexten zufolge finden diese Formen reichlich Verwendung als Adverbiale, die neben den Nominativformen die zeitliche Dauer ausdrücken, und zwar in dem Gebiet der Ostdialekte, das die Mundarten von P, B, M und U umfasst, westlicher jedoch nicht. Einige Beispiele: šordâm šüšşâlam-at kum $i$ (od. $k u m$

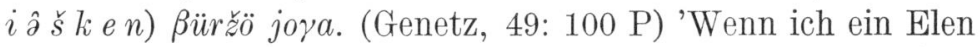
schlachte, fliesst dessen Blut drei Jahre lang.' / lu $t^{\prime} \dot{s} k 0$

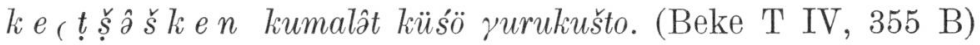
'Man opfert fünfzehn Tage lang auf dem Opferberg.' / $i k$ arńa šk e n âštat šor (t)-d'olâm mariįlak. (Beke T IV, 34 M) 'Die Tscheremissen feiern (eig. halten) (die) Weihnachten eine

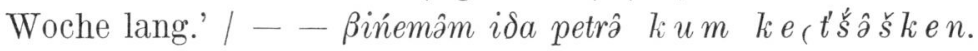
(Beke M, 458: 4-5 U) '- - die Grube schüttet drei Tage lang nicht zu.' - - kandram punat ta šukâšken. (Beke T III, 320 U) ' - - sie flechten das Seil lange.'

Ausser der zeitlichen Dauer kann durch auf -šken endende Formen jedoch auch die Terminativität ausgedrückt werden. Diese Bedeutung kommt den pronominalstämmigen auf -šken ausgehenden Formen zu. Sie begegnen auch im Wiesendialekt, ausserhalb des obengenannten Gebietes also. Einige Belege: ̌̌uarńan tolźa! tuške n u pörteš oltena. (Beke M, 183: 5 U) 'Kommet Sonntags! Bis dahin heizen wir im neuen Hause ein.' / ku us k e n kodat olaš? U, C / ku usk e n olaš kodat? M (Wichmann $R, 429$ ) 'Bis wielange bleibst du in der Stadt?' Die Formen kušken und tušken gehören auch zur östlichen 
Schriftsprache (s. z.В. Марийско-русский словарь, 254 und 603).

In den westdialektalen Varianten des obenzitierten Beispielsatzes von Wichmann begegnet eine auf -škeßek ausgehende

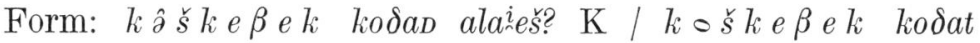
alaš? JL id. Die Formen kâškeßek 'bis wohin, bis wann', tiškeßek 'bis hierher, bis jetzt' und taškeßek 'bis dahin' gehören nach dem genannten Wörterbuch ebenfalls zur westlichen Schriftsprache.

Von denselben Pronomina lassen sich dialektweise noch andere Formen mit gleicher Bedeutung bilden. In Ramstedts Wörterbuch des Westdialekts z.B. findet sich neben der Form kâškeßek die Lautgestalt kâškeße sowie entsprechend neben der Form taškeßek die Form taškeße; ferner habe ich die Form tâškeße auch aus einem Text der östlichen Schriftsprache verzeichnet: $T$ ви и кев в куито лийынат? (Ончыко 6/1963, 15) 'Wo warst du bis jetzt?' Weiterhin können die von Reguly aus dem Wiesendialekt aufgezeichneten Lautgestalten kuškeven und kuškeneš erwähnt werden, z.B. $k u s ̌ k$ even $($ od. kušken) tin-dened ila? (Budenz NyK 3, 118 C) 'Wie lange wohnt er bei dir?' und $k u s ̌ k$ ene $s$ kodad olaš? (Budenz NyK 3, 118 C) 'Bis wann bleibst du in der Stadt?'; in der letztgenannten Form ist dem kušken noch deutlich die Lativendung -eš angefügt.

Alle diese pronominalstämmigen Formen sind also terminativ. Es ist offenbar, dass in ihnen allen die Illativendung -ške enthalten ist, mit der ein oder einige Morpheme verbunden wurden, die vielleicht die terminative Bedeutung der Form forcierten. Auf den ersten Blick scheint die Angelegenheit verhältnismässig klar zu sein: an die Illativendung wäre das Suffixelement $-\beta e k$ oder $-\beta e n$ getreten, das einen $k$-Lativ oder einen $n$-Lativ enthält und offensichtlich auf ein selbständiges Wort $\beta$ el 'Seite' zurückgeht (s. z.В. И. С. Галкин, Историческая грамматика марийского языка, морфология, S. 187). Später wäre dann das unbetonte $\beta$ dialektweise geschwunden, und somit wäre das Suffix -šken entstanden.

Ein Umstand warnt jedoch vor allzu geradlinigen Schlüssen, und das ist die Qualität des Vokals, der dem Element - $\beta e k$ 
oder - ßen vorangeht. Hätte sich die Entwicklung so vollzogen wie oben skizziert, würde man erwarten, dass der betreffende Vokal reduziert ist; in allen von mir verzeichneten Beispielen ist es jedoch ein volles $e$. Somit besteht denn auch die Möglichkeit, dass der Typus kušken ursprünglicher ist; das Auslaut-en darin ist vielleicht zusammenzustellen mit dem in einigen anderen Partikeln, u.a. im terminativen marken, matken usw. auftretenden en-Auslaut. Wie marken und viele andere auf -en ausgehende Partikeln dialektweise in der $n$-losen Gestalt marke., matke vorkommen (die Betonung auf dem letzten $e^{-V}$-Vokal des Wortes zeigt, dass danach ein Konsonant geschwunden ist), hatte vielleicht auch der Typus kušken die Tendenz, dialektal ohne $n$ aufzutreten. Weil er dann aber in zahlreichen Dialekten der Form nach dem (nur anders betonten) Illativ gleich geworden wäre, verlangte vielleicht das Sprachgefühl die Hinzufügung einer verstärkenden Partikel; diese Partikel wird in der heutigen Sprache sichtbar als Auslaut - $\beta e k,-\beta e n$ und $-\beta e$.

Jedenfalls sieht es so aus, als sei die ursprüngliche Bedeutung des Suffixes -šken terminativ gewesen; diese Bedeutung hat sich in den pronominalstämmigen Formen erhalten. In einem ausgedehnten Gebiet der Ostdialekte hat sich die ursprüngliche Bedeutung jedoch erweitert: indem es an Wörter trat, die eine Zeitangabe ausdrücken, erhielt das Suffix eine die Dauer des Geschehens kennzeichnende Bedeutung. Eigentlich wurde das Morphem -šken in diesen Mundarten direkt zu einer Art temporaler Kasusendung.

Es ist eine allgemeine Erscheinung, dass eine Form mit ursprünglich terminativer Bedeutung auch die Zeitdauer ausdrücken kann. Eine aufschlussreiche Parallele findet sich u.a. im Ungarischen, wo das Suffix -ig beide Bedeutungen hat, was mitunter sogar als störend empfunden werden kann: két óráig bedeutet 'bis zwei Uhr', kann aber auch 'zwei Stunden [lang]' heissen (s. z.B. A mai magyar nyelv rendszere. Leíró nyelvtan II, S. 194).

Im Tscheremissischen selbst aber gibt es neben dem Element -šken auch einige Postpositionen, die einerseits eine terminative, andererseits eine die zeitliche Dauer einer Handlung oder eines Geschehens anzeigende Bedeutung haben können. In den 
östlichsten Mundarten kann man mit Hilfe der Terminativpostposition marke, matke, marken, martene usw. eine Konstruktion bilden, die in genau denselben Satzzusammenhängen begegnet wie die zu Beginn dieses Aufsatzes behandelte Nominativform, die die zeitliche Dauer ausdrückt. Einige Beispiele: $n$ ə l bič ke č e marten üdar tušto aškanže küleš nastam burgemam urgen jamdəla. (Sebeok - Ingemann, 76 B) 'Vier fünf Tage fertigt das Mädchen dort nähend die Sachen und Kleider

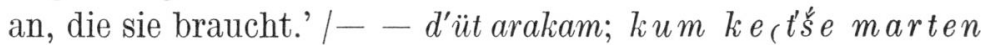
$d^{\prime} \ddot{u} t$. (Beke T III, $176 \mathrm{U}$ ) '- - [sie] trinken Branntwein; sie trinken drei Tage hindurch.' / modât šuko martene. (Beke T III, 187 U) 'Sie spielen lange.' Über die terminative Verwendung der Postposition finden sich Beispiele in dem Aufsatz von Julius Mägiste, der in der Veröffentlichung Verba docent steht, S. S. $128-129$.

Zu den etwas weiter westlicher gesprochenen Mundarten gehört die genau entsprechende Verwendung der Terminativpostposition jotke, jakte usw.; einige Belege: $k \circ m l$ o $\gamma$ e t $s e$ d jok te ki $i^{i} a$ kotko-šuešte. (Beke TT, 71:219 JT) 'Dreissig Tage liegen [die Frösche] im Ameisenhaufen.' / $k$ ol $l o] \quad i$ djokte ere djolen sorta-tol - - (Beke TM, 172 JT) 'Zwanzig Jahre brannte die Kerzenflamme immerfort - - .' Zum terminativen Gebrauch der Postposition s. Mägistes erwähnten Aufsatz (Verba docent, S. 129). Es ist offenbar, dass der Sprachträger durchaus nicht in allen Fällen jene Adverbiale der Zeit voneinander unterscheidet, mittels derer ein Zeitabschnitt ausgedrückt wird, währenddessen etwas geschieht, sowie jene, die die zeitliche Dauer des Geschehens angeben. So trifft man eine mit der Postposition rodâm 'während' gebildete Konstruktion mitunter in einem Satzzusammenhang an, wo ebenso gut z.B. eine die zeitliche Dauer ausdrückende Nominativ-

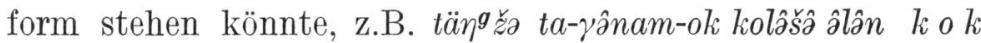

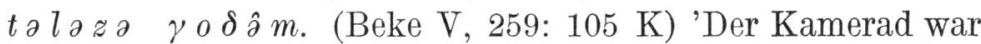
damals etwa seit zwei Monaten tot.' / $k \circ m k e$ c $t s$ a $\gamma \circ \delta \hat{a} m$ jüte katste šalyâšna. (Beke T I, 15 JO) 'Drei Tage lang blieben (eig. standen) wir ohne Speise und Trank.' Es sei bemerkt, dass es sich in diesen Ausdrücken nicht um eine objektartige Bestimmung handelt, auch wenn yodâm der Form nach ein 
Akkusativ ist; in jenen Dialekten, aus denen die genannten Belege stammen, steht das Adverbiale der Zeitangabe nämlich stets in der Nominativform.

In einigen Satzzusammenhängen kann der Sprecher des Tscherem. anstelle eines Adverbiale der Zeitbestimmung in Nominativ- oder Akkusativform auch zahlreiche andere Postpositionskonstruktionen wählen. Die in Frage kommenden Postpositionen sind z.B. ßošt 'durch', yotš 'durch' und mutško 'auf', z.B. miń erkân ü $t \beta$ ošt lištâšâm. (Beke M, 182: 1 U) 'Ich machte das langsam, die ganze Nacht.' / telam šim mužo

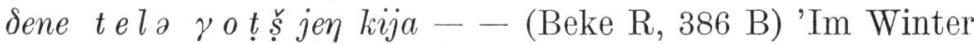
liegt der Mann den ganzen Winter in der schwarzen Krankheit - -' I marâ roßotaja ke(tšs mât škâ. (Beke V, 302: 4 K) 'Der Mann arbeitet den ganzen Tag.' Diese Postpositionsausdrücke lassen sich jedoch mit den früher behandelten nicht völlig gleichstellen. Thre Anwendung scheint nämlich stark eingeschränkt zu sein: die Postpositionen stehen nur in Verbindung mit einigen bestimmten, die Zeit ausdrückenden Wörtern. Andererseits lässt sich in den Bedeutungen dieser Ausdrücke eine gewisse zusätzliche Nuance feststellen, die in den deutschen Übersetzungen der Texte oft z.B. durch das Wort 'ganz' wiedergegeben wird.

Als Beweis dafür, wie leicht die Konstruktionen, in denen zeitliche Verhältnisse ausgedrückt werden, von einer Bedeutung zur anderen übergehen, sei abschliessend ein Beispiel angeführt, wo die $\beta$ ošt-Postpositionskonstruktion ausnahmsweise einen Zeitpunkt ausdrückt, in dessen Verlauf etwas geschieht:

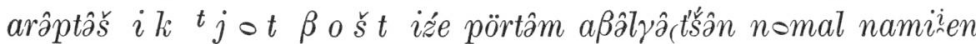
djer tṇreš šânda. (Beke T I, 585 C) 'Der arâptâš bringt in der (eig. einer) Nacht ein kleines Haus aus dem Dorf und stellt es ans Ufer des Teiches.'

Almo Alhoniemi 